\title{
Thinking on Transcranial Direct Current Stimulation (tDCS) in Reading Interventions: Recommendations for Future Research Directions
}

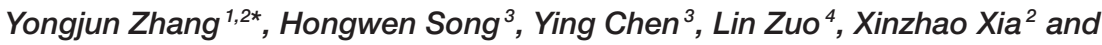 \\ Xiaochu Zhang ${ }^{2,5,6,7 *}$ \\ ${ }^{1}$ School of Foreign Languages, Anhui Jianzhu University, Hefei, China, ${ }^{2}$ Centers for Biomedical Engineering, School of \\ Information Science and Technology, University of Science and Technology of China, Hefei, China, ${ }^{3}$ School of Humanities \\ and Social Science, University of Science and Technology of China, Hefei, China, ${ }^{4}$ CAS Key Laboratory of Brain Function \\ and Disease, School of Life Science, University of Science and Technology of China, Hefei, China, ${ }^{5}$ Hefei Medical Research \\ Center on Alcohol Addiction, Anhui Mental Health Center, Hefei, China, ${ }^{6}$ Academy of Psychology and Behavior, Tianjin \\ Normal University, Tianjin, China, ${ }^{7}$ Hefei National Laboratory for Physical Sciences at the Microscale and School of Life \\ Sciences, University of Science and Technology of China, Hefei, China
}

Keywords: reading difficulties, tDCS, reading interventions, opinion, future directions

\section{INTRODUCTION}

OPEN ACCESS

Edited by:

Hasan Ayaz,

Drexel University, United States

Reviewed by: Nathaniel Bridges, Air Force Research Laboratory,

United States

Helena Knotkova MJHS Institute for Innovation in Palliative Care, United States

*Correspondence: Yongjun Zhang andyzhyj@126.com

Xiaochu Zhang

zxcustc@ustc.edu.cn

Received: 04 October 2018 Accepted: 25 April 2019 Published: 22 May 2019

Citation:

Zhang Y, Song H, Chen Y, Zuo L, Xia X and Zhang $X$ (2019) Thinking on Transcranial Direct Current Stimulation (tDCS) in Reading Interventions:

Recommendations for Future Research Directions.

Front. Hum. Neurosci. 13:157. doi: 10.3389/fnhum.2019.00157
Transcranial direct current stimulation (tDCS) is a non-invasive neural modulation technique to remediate many neural deficits. tDCS involves the application of a direct current (1-2 mA) which modulates the resting membrane potential of cortical neurons (Dasilva et al., 2011; Brunoni et al., 2012). In tDCS, anodal stimulation results in subthreshold depolarization and increases the likelihood of neurons' firing, while cathodal stimulation hyperpolarizes neurons and decreases the likelihood of their firing (Nitsche et al., 2003; Stagg and Nitsche, 2011). The outlasting neuroplastic effects of tDCS depend on synaptic plasticity of glutamatergic neurons (Liebetanz et al., 2002; Nitsche et al., 2003). Furthermore, magnetic resonance spectroscopy (MRS) studies have shown reduction in gamma-aminobutyric acid (GABA) and increase in glutamate following tDCS (Rae et al., 2009; Stagg et al., 2009), and tDCS can modulate postsynaptic connectivity within brain networks (Stagg and Nitsche, 2011; Meinzer et al., 2014). Therefore, it is thought that the long-term effects of tDCS may share common characteristics with long-term depression (LTD) and long-term potentiation (LTP) in the regulation of neuroplasticity (Elmasry et al., 2015).

Functional imaging studies have converged in identifying a left-hemisphere lateralized reading network, including the inferior frontal gyrus, the occipito-temporal region, and the parietotemporal region (Gabrieli, 2009; Richlan et al., 2010, 2011; Martin et al., 2015). Atypical activations of these regions in reading tasks have been reported in individuals having reading difficulties (Horwitz et al., 1998; Paulesu et al., 2001; Mccandliss and Noble, 2003; Hoeft et al., 2007; Morken et al., 2017).

In view of the neural modulation property of tDCS and the benefits of applying tDCS to modulating reading efficiency in healthy subjects (Antal et al., 2014; Thomson et al., 2015), several studies have adopted tDCS in reading interventions among the individuals with developmental dyslexia (DD) and below-average readers (Turkeltaub et al., 2012; Heth and Lavidor, 2015; Costanzo et al., 2016a,b, 2018; Younger et al., 2016). These studies mainly follow the traditional assumption that anodal/cathodal stimulation increases/decreases cortical neural excitability, and in these studies, the anodal/cathodal electrodes $(5 \times 5 \mathrm{~cm}$ or $5 \times 7 \mathrm{~cm})$ were placed over the targeted areas generally set within the temporo-parietal cortex. However, the observed enhancement of specific reading abilities (mainly including grapheme-to-phoneme mapping, phonological 
processing, reading efficiency, rhyming judgment) was inconsistent across studies, and in some cases the effect sizes of the same outcome measure were quite different between studies (Turkeltaub et al., 2012; Younger et al., 2016). Furthermore, due to the distributed feature of traditional tDCS and the fact that no neural evidence was reported in the results of existing reading-remediation studies, we cannot rule out the possibility that the outcomes may also in part arise from the stimulation of regions adjacent to the target area. Therefore, we herein put forth our opinion on issues concerning stimulation parameters, populations, defocusing, combination of tDCS with cognitive training, and outcome measures, which may influence the evaluation of tDCS-based reading interventions and should be considered more carefully in future studies. Both dyslexics and readers below the average reading level are categorized as individuals with reading difficulties in this paper.

\section{STIMULATION PARAMETERS}

The variable and inconsistent findings of tDCS-based reading interventions may relate closely to some stimulation parameters, including current intensity, number of sessions, and session duration. Current intensities of $1 \mathrm{~mA}$ (Costanzo et al., 2016a,b, 2018) or 1.5 mA (Turkeltaub et al., 2012; Heth and Lavidor, 2015; Younger et al., 2016) were used in available studies, and current density varied between 0.04 and $0.06 \mathrm{~mA} / \mathrm{cm}^{2}$. Although higher current density may yield larger cognitive effects (Boggio et al., 2006; Teo et al., 2011), it should be adopted with caution in that higher density may cause skin burns (Palm et al., 2008), interfere with double blinding (O'Connell et al., 2012), and influence activities in regions deeper than those to be targeted (De Aguiar et al., 2015). We suggest that future tDCS-based reading interventions use more randomized controlled trials (RCTs) to obtain an optimal balance between stimulation intensity and intervention effect.

Number of sessions is another critical issue that may influence tDCS effect. Two review studies found no evidence of a reliable effect of single-session tDCS on cognitive performance in healthy participants (Horvath et al., 2015; Westwood and Romani, 2017), and another systematic review suggested that tDCS comprising multiple sessions can ameliorate symptoms of several psychiatric disorders (Kekic et al., 2016). Therefore, it is also worth our investigation to figure out a more feasible number of tDCS sessions among people with reading difficulties. However, the variable results of single session (Turkeltaub et al., 2012; Costanzo et al., 2016b; Younger et al., 2016) or multiplesession tDCS-based reading interventions (Heth and Lavidor, 2015; Costanzo et al., 2016a, 2018) were not expounded from the perspective of this issue. In view of the limited studies within the scope under discussion, more studies using between-subject design or just replicating prior work are required to draw a general conclusion on the issue of session number.

The studies under discussion didn't vary too much regarding the parameter of session duration, and 20 min was a common adoption. Given that relatively longer durations (above $10 \mathrm{~min}$ ) determine larger effects (Hill et al., 2016; Giulia et al.,
2019), 20 min duration can be still feasible in future reading interventions. Duration between 10 and $20 \mathrm{~min}$, for example, $15 \mathrm{~min}$, may be preferable if the effect is not inferior to that with $20 \mathrm{~min}$. As for those studies consisting of several stimulation sessions (Heth and Lavidor, 2015; Costanzo et al., 2016b, 2018), interval between sessions (IBS) is also an important factor worth consideration. Research has shown that the IBS may influence tDCS outcome (Monte-Silva et al., 2010), and the inhibitory effect of cathodal-tDCS was delayed while the excitatory effect of anodal-tDCS converted into inhibition with a 24-h IBS (MonteSilva et al., 2013). Based on available findings, it is suggested that multiple sessions with a daily frequency and a 1-week washout period seem to be suitable for populations with language disorder (De Aguiar et al., 2015). Future tDCS-based reading interventions need to compare the effects of tDCS with different IBS, especially for those studies using crossover design in which carry-over effect is more easily to arise.

\section{FEATURES OF DIFFERENT GROUPS OF POPULATIONS}

tDCS effects may vary between healthy participants and individuals with cognitive or neuropsychiatric disorders (Ferrucci et al., 2009; Brunoni and Vanderhasselt, 2014; Dedoncker et al., 2016). Furthermore, in two studies exploring tDCS modulatory effect on participants' reading performance, both Thomson et al. (2015) and Westwood et al. (2017) suggested that tDCS are more likely to induce effect in brains with more dysfunctional neuronal excitability, and there is less space for further improvement in already optimized brain areas. Existing tDCS-based reading interventions among dyslexics or below-average readers are more or less effective, but few of them take this issue into account in the interpretation of their results. It is possible that the lesion state or severity level of the reading-related areas is a continuum, thus future research can examine whether different stimulation dose should be applied to modulate reading efficiency in participants ranging from below-average readers to dyslexics. Notably, behavioral training may be a more feasible alternative intervention for those low-toaverage readers, given that tDCS may disrupt inter-hemispheric inhibition in low-to-average readers and induced a negative effect on their phonological working memory abilities, as is shown by Younger et al. (2016).

Besides, the atypical brain areas of dyslexic populations may also vary with their different cultures and ages. Chinese dyslexic individuals exhibit atypical activation in the left prefrontal cortex (Siok et al., 2004), while French, Italian, and English readers with dyslexia exhibit hypoactivation in the left temporal cortex (Paulesu et al., 2001). Although there is a dysfunction of a left ventral occipito-temporal region in both children and adults, underactivation in bilateral inferior parietal regions was only found in children and underactivation in superior temporal cortex was only found in adults (Richlan et al., 2011). Therefore, future tDCS-based reading interventions are supposed to set different target areas and use different electrode montages in subjects from different cultures and age groups. 


\section{DEFOCUSING}

The reduction of spatial focality and modulation efficiency at the targeted areas during tDCS is defined as defocusing, which is one notable limitation of the traditional tDCS protocol using 10-20 EEG notation (Datta et al., 2008; Boggio et al., 2009; Thomson et al., 2015). The low conductivity of skull, the electric current concentration on the edge of gyri, and the widespread excitatory effect of the anodal tDCS (Datta et al., 2009; Thomson et al., 2015) are believed to be the leading factors resulting in defocusing. Computational head models have been developed to predict a more focal directional effect of current flow under the electrodes (Wagner et al., 2007; Datta et al., 2009; Sadleir et al., 2010; Turkeltaub et al., 2012). A computational head model is a structural magnetic resonance imaging/MRI-derived finite element model of an adult's head with a high resolution $\left(1 \mathrm{~mm}^{3}\right)$, and the head is segmented into different compartments representing brain tissues (Datta et al., 2009). The finite element mesh generated from the segmented data can be exported to COMSOL Multiphysics, a finite element software package, for the computation of electric fields and the simulation of electrode montage (Datta et al., 2009; Bai et al., 2014). Computational models have been used to develop electrode configurations, and a $4 \times 1$ ring configuration has been shown to enhance focality by positioning a small central electrode surrounded by four return electrodes, in contrast to conventional tDCS using two large rectangular pads (Datta et al., 2009; Kuo et al., 2013; Gbadeyan et al., 2016). This ring configuration is defined as high-definition tDCS (HD-tDCS) and has been demonstrated to restrict upwards of $30 \%$ of the stimulation peak within the ring perimeter (Edwards et al., 2013).

Computational models are rarely developed to guide the tDCS current flow in reading remediation. A recent study investigated the effect of $\mathrm{tDCS}$ on reading abilities of children and adolescents with dyslexia, but found no positive effects on text reading and high-frequency words (Costanzo et al., 2016b). It is likely that the defocusing of the traditional tDCS utilized in this study led to the negative result with regard to grapheme identification, which is also implied by Costanzo and collaborators. Another study selected the left inferior parietal lobe (IPL) as the targeted area and measured low-to-average readers' improvement on both rhyming judgment and single word reading efficiency (Younger et al., 2016). Results showed that left IPL stimulation relatively "impairs" participants' performance on the rhyming judgment task, even though it resulted in gains in the other task. In fact, it is the left IPL that underlies the storage of short-term phonological information and verbal working memory (Ravizza et al., 2002, 2004; Yue et al., 2018) needed for the rhyming judgment task, so we propose that the unexpected effect on the rhyming task could also be partially due to the defocusing in the experiment. Consequently, similar to the study conducted by Costanzo and collaborators, the current may diffuse to adjacent parietal areas so that the unexpected result emerged. In contrast, below-average readers demonstrated improved word reading efficiency as expected by Turkeltaub et al. (2012). The authors attributed this result to a finite head model generated in the study for the computation of electric fields, which predicted a focal effect under the electrodes centering over left posterior temporal cortex (pTC) and right pTC. But the above-mentioned HD-tDCS was not adopted, which might partly explain why there was no tDCS effect on nonword reading efficiency in this study.

To date, only a few studies made a direct comparison between the effect of conventional tDCS and that of HD-tDCS, and most of these studies concluded that the effects of HD-tDCS are at least comparable to that of conventional tDCS. Notably, plastic changes in the primary motor cortex showed a more delayed peak at $30 \mathrm{~min}$ and longer lasting after-effects after HD-tDCS, as compared to conventional tDCS (Kuo et al., 2013). Besides, in a study aiming at remediating aphasia, change in accuracy of trained items was found to be numerically higher (although not statistically significant) for HD-tDCS compared to conventional tDCS for most patients (Richardson et al., 2015). In the light of these positive findings, HD-tDCS has its potential to be realized in future reading interventions and achieve greater gains for both dyslexics and below-average readers.

\section{COMBINING TDCS WITH READING-RELATED COGNITIVE TRAINING (CT)}

When tDCS sessions are given in combination with CT or other rehabilitation protocols for improving motor or cognitive performance, better outcomes are achieved than with the CT or standard rehabilitation alone (Lindenberg et al., 2010; Fridriksson et al., 2011; Ditye et al., 2012; Martin et al., 2013; Penolazzi et al., 2015; Lawrence et al., 2018). The principle underlying such combination protocol is that transcranial electric stimulation (tES) can enhance the synaptic transmission and strength in neural pathways activated by the CT, so that the endogenous activation (CT) and exogenous neuromodulation (tES) can work together to facilitate the activation of neural networks subserving cognitive functions (Miniussi and Vallar, 2011; Elmasry et al., 2015). As such, we think that the efficacy of reading interventions may also be enhanced by combining tDCS with reading-related CT or tasks.

Since the CT employed in the intervention obviously cannot encompass all the different reading tasks subjects may be faced with in everyday life, it is worth further research whether the effects of combining tDCS with CT can be transferred to nontrained tasks. Given the essential role of working memory (WM) in reading ability (Hoeft et al., 2007; Pham and Hasson, 2014), several studies investigating the effects of combining tDCS with CT on the WM and transfer effects on non-trained WM tasks may shed light on this issue. The active tDCS + CT group did show significantly greater gains than the tDCS-only group or the CT-only group in non-trained WM tasks (Martin et al., 2013; Richmond et al., 2014), but no significant outcome differences were found between the active tDCS + CT group and the sham tDCS + CT group. While another study demonstrated that in a non-trained WM task, the active tDCS + CT group performed significantly better compared to the sham tDCS + CT group (Park et al., 2013). This result may arise from the electrode montage that anodes were attached to bilateral prefrontal cortex 
stimulated by two stimulators and cathodes were attached to the non-dominant arm. Such a montage corresponded with the notion that the left dorsolateral prefrontal cortex (dlPFC) is responsible for verbal WM while the right dlPFC subserves spatial WM (Ruf et al., 2017). Both verbal and spatial memory are correlated with reading comprehension (Swanson and Howell, 2001; Pham and Hasson, 2014), and thus the dose of treatment were doubled.

Consequently, a combination treatment protocol can be feasible in treating reading difficulties, and unilateral tDCS combined with CT can render a transfer effect; notably, bilateral tDCS is more preferable if WM-related trainings are administered. These propositions warrant future systematic exploration. Other factors such as the timing of tDCS relative to $\mathrm{CT}$, frequency of $\mathrm{tDCS}+\mathrm{CT}$ sessions, the type of population targeted (below-average readers or individuals with DD) may also influence the combination treatment outcome (Elmasry et al., 2015; Cancer and Antonietti, 2018).

\section{ADDING OUTCOME MEASURES BY USING NEUROIMAGING METHODS}

Adopting behavioral remediation or instructional treatment for reading difficulties have been documented as being able to improve brain activations (Temple et al., 2003; Simos et al., 2007; Meyler et al., 2008; Richards and Berninger, 2008). However, behavioral results are mainly reported as the outcome reading measures in the existing studies of tDCS-based reading remediation. Given that tDCS is a technique modulating neural activity, we propose that neuroimaging techniques such as electroencephalography (EEG), fMRI, functional near infrared spectroscopy (fNIRS) should be used to explore the neural activity changes after tDCS remediation.

Evidence has shown EEG + tDCS, fMRI+ tDCS, or fNIRS + tDCS can be used to monitor tDCS-induced changes of the neural activities involved in sustained attention (Miller et al., 2015), semantic processing (D’Mello et al., 2017), and spatial working memory (McKendrick et al., 2015), all of which have also been found to be impaired in the individuals with reading difficulties (Schulz et al., 2008; Pham and Hasson, 2014; Staels and Van den Broeck, 2017). As such, those neuroimaging methods can also be integrated into future tDCS-based reading interventions to detect the potential neural changes, which can be taken as an additional outcome measure. Apart from the above-mentioned potential neural changes, future studies can try to investigate whether other reported atypical cortical band activations (Sklar et al., 1972; Fein et al., 1986; Spironelli et al., 2008; Penolazzi et al., 2010; Papagiannopoulou and Lagopoulos, 2016), event-related potentials (ERPs) (Horowitz-Kraus, 2016), and brain activations (for a review, see Gabrieli, 2009) in populations with reading difficulties can be normalized after tDCS-based interventions.

\section{CONCLUSIONS}

Currently, the field of tDCS-based reading interventions is still at its infancy and the related literature is relatively sparse compared to other tDCS-based research. It is therefore too early to conclude that tDCS is generally effective in this field, and we should have an objective assessment of the limitations of this technique and of the conditions in which there is limited or even negative effect. In this opinion article, we have put forth some recommendations that merit more attention in future tDCSbased reading interventions. Further evidence from larger-scale RCTs is especially required for those dyslexic individuals so as to define reproducible stimulation parameters for certain groups of samples. Based on empirical evidence and safe ethical grounds (Kekic et al., 2016), tDCS is expected to be a promising device in reading interventions, especially when it is given in combination with CT and neuroimaging methods.

\section{AUTHOR CONTRIBUTIONS}

$\mathrm{YZ}$ wrote the article. XZ revised each draft of the article. YZ, $\mathrm{XZ}$, and HS helped to conceive the opinion. YC, LZ, XX and XZ contributed to data collection.

\section{FUNDING}

This work was supported by grants from the National Key Basic Research Program (2016YFA0400900 and 2018YFC0831101), the National Natural Science Foundation of China (31471071, 31771221, 61773360, and 71874170), the Fundamental Research Funds for the Central Universities of China, the National Social Science Foundation of China (18BYY015), the Humanities and Social Science Research Foundation of Education Department of Anhui Province (SK2019A0661, SK2018A0571, and XJ2018002002), and the Teaching Research Foundation of Anhui Province (2015JYXM244 and 2016JYXM0216).

\section{REFERENCES}

Antal, A., Ambrus, G. G., and Chaieb, L. (2014). Toward unraveling reading-related modulations of tDCS-induced neuroplasticity in the human visual cortex. Front. Psychol. 5:642. doi: 10.3389/fpsyg. 2014.00642

Bai, S., Dokos, S., Ho, K. A., and Loo, C. (2014). A computational modelling study of transcranial direct current stimulation montages used in depression. Neuroimage 87, 332-344. doi: 10.1016/j.neuroimage.2013. 11.015
Boggio, P. S., Ferrucci, R., Rigonatti, S. P., Covre, P., Nitsche, M., PascualLeone, A., et al. (2006). Effects of transcranial direct current stimulation on working memory in patients with parkinson's disease. J. Neurol. Sci. 249, 31-38. doi: 10.1016/j.jns.2006.05.062

Boggio, P. S., Fregni, F., Valasek, C., Ellwood, S., Chi, R., and Gallate, J., et al. (2009). Temporal lobe cortical electrical stimulation during the encoding and retrieval phase reduces false memories. PLoS ONE 4:e4959. doi: 10.1371/journal.pone.0004959

Brunoni, A. R., Nitsche, M. A., Bolognini, N., Bikson, M., Wagner, T., and Merabet, L., et al. (2012). Clinical research with transcranial direct current 
stimulation (tDCS): challenges and future directions. Brain Stimul. 5, 175-195. doi: 10.1016/j.brs.2011.03.002

Brunoni, A. R., and Vanderhasselt, M. A. (2014). Working memory improvement with non-invasive brain stimulation of the dorsolateral prefrontal cortex: a systematic review and meta-analysis. Brain Cogn. 86, 1-9. doi: 10.1016/j.bandc.2014.01.008

Cancer, A., and Antonietti, A. (2018). tDCS modulatory effect on reading processes: a review of studies on typical readers and individuals with dyslexia. Front. Behav. Neurosci. 12:162. doi: 10.3389/fnbeh.2018.00162

Costanzo, F., Rossi, S., Varuzza, C., Varvara, P., Vicari, S., and Menghini, D. (2018). Long-lasting improvement following tDCS treatment combined with a training for reading in children and adolescents with dyslexia. Neuropsychologia. 2018:016. doi: 10.1016/j.neuropsychologia.2018.03.016

Costanzo, F., Varuzza, C., Rossi, S., Sdoia, S., Varvara, P., and Oliveri, M., et al. (2016a). Reading changes in children and adolescents with dyslexia after transcranial direct current stimulation. Neuroreport 27, 295-300. doi: 10.1097/WNR.0000000000000536

Costanzo, F., Varuzza, C., Rossi, S., Sdoia, S., Varvara, P., and Oliveri, M., et al. (2016b). Evidence for reading improvement following tDCS treatment in children and adolescents with dyslexia. Restor. Neurol. Neurosci. 34:215. doi: 10.3233/RNN-150561

Dasilva, A. F., Volz, M. S., Bikson, M., and Fregni, F. (2011). Electrode positioning and montage in transcranial direct current stimulation. J. Visual. Exp. 51:e2744. doi: $10.3791 / 2744$

Datta, A., Bansal, V., Diaz, J., Patel, J., Reato, D., and Bikson, M. (2009). Gyriprecise head model of transcranial direct current stimulation: improved spatial focality using a ring electrode versus conventional rectangular pad. Brain Stimul. 2, 201-207.e1. doi: 10.1016/j.brs.2009.03.005

Datta, A., Elwassif, M., Battaglia, F., and Bikson, M. (2008). Transcranial current stimulation focality using disc and ring electrode configurations: FEM analysis. J. Neural Eng. 5, 163-174. doi: 10.1088/1741-2560/5/2/007

De Aguiar, V., Paolazzi, C. L., and Miceli, G. (2015). TDCS in poststroke aphasia: the role of stimulation parameters, behavioral treatment and patient characteristics. Cortex 63, 296-316. doi: 10.1016/j.cortex.20 14.08 .015

Dedoncker, J., Brunoni, A. R., Baeken, C., and Vanderhasselt, M. A. (2016). A systematic review and meta-analysis of the effects of transcranial direct current stimulation (tDCS) over the dorsolateral prefrontal cortex in healthy and neuropsychiatric samples: influence of stimulation parameters. Brain Stimul. 9, 501-517. doi: 10.1016/j.brs.2016.04.006

Ditye, T., Jacobson, L., Walsh, V., and Lavidor, M. (2012). Modulating behavioral inhibition by tDCS combined with cognitive training. Exp. Brain Res. 219, 363-368. doi: 10.1007/s00221-012-3098-4

D'Mello, A. M., Turkeltaub, P. E., and Stoodley, C. J. (2017). Cerebellar tdcs modulates neural circuits during semantic prediction: a combined tDCS-fMRI study. J. Neurosci. 37, 1604-1613. doi: 10.1523/JNEUROSCI.28 18-16.2017

Edwards, D., Cortes, M., Datta, A., Minhas, P., Wassermann, E. M., and Bikson, M. (2013). Physiological and modeling evidence for focal transcranial electrical brain stimulation in humans: a basis for high-definition tDCS. Neuroimage 74, 266-275. doi: 10.1016/j.neuroimage.2013.01.042

Elmasry, J., Loo, C., and Martin, D. (2015). A systematic review of transcranial electrical stimulation combined with cognitive training. Restor. Neurol. Neurosci. 33, 263-278. doi: 10.3233/RNN-140473

Fein, G., Galin, D., Yingling, C. D., Johnstone, J., Davenport, L., and Herron, J. (1986). EEG spectra in dyslexic and control boys during resting conditions. Electroencephalogr. Clin. Neurophysiol. 63, 87-97. doi: 10.1016/0013-4694(86)90001-5

Ferrucci,R., Bortolomasi, M., Brunoni, A. R., Vergari, M., Tadini, L., Giacopuzzi, M., et al. (2009). Comparative benefits of transcranial direct current stimulation (tDCS) treatment in patients with mild/moderate vs. severe depression. Clin. Neuropsychiatry 6:246e251.

Fridriksson, J., Richardson, J. D., Baker, J. M., and Rorden, C. (2011). Transcranial direct current stimulation improves naming reaction time in fluent aphasia a double-blind, sham-controlled study. Stroke 42, 819-821. doi: 10.1161/STROKEAHA.110.600288

Gabrieli, J. D. (2009). Dyslexia: a new synergy between education and cognitive neuroscience. Science 325, 280-283. doi: 10.1126/science.1171999
Gbadeyan, O., Steinhauser, M., Mcmahon, K., and Meinzer, M. (2016). Safety, tolerability, blinding efficacy and behavioural effects of a novel MRI-compatible, high-definition tDCS set-up. Brain Stimul. 9, 545-552. doi: 10.1016/j.brs.2016.03.018

Giulia, G., Miguel, A. V., Miroslav, S., Matteo, F., and Angela, M. (2019). A systematic review and meta-analysis of the effects of transcranial direct current stimulation (tDCS) on episodic memory Brain Stimul. 12, 231-241. doi: 10.1016/j.brs.2018.11.008

Heth, I., and Lavidor, M. (2015). Improved reading measures in adults with dyslexia following transcranial direct current stimulation treatment. Neuropsychologia 70, 107-113. doi: 10.1016/j.neuropsychologia.2015.02.022

Hill, A. T., Fitzgerald, P. B., and Hoy, K. E. (2016). Effects of anodal transcranial direct current stimulation on working memory: a systematic review and metaanalysis of findings from healthy and neuropsychiatric populations. Brain Stimul. 9, 197-208. doi: 10.1016/j.brs.2015.10.006

Hoeft, F., Meyler, A., Hernandez, A., Juel, C., Taylorhill, H., Martindale, J. L., et al. (2007). Functional and morphometric brain dissociation between dyslexia and reading ability. Proc. Natl. Acad. Sci. U.S.A. 104, 4234-4239. doi: 10.1073/pnas.0609399104

Horowitz-Kraus, T. (2016). Improvement of the error-detection mechanism in adults with dyslexia following reading acceleration training. Dyslexia 22, 173-189. doi: 10.1002/dys.1523

Horvath, J. C., Forte, J. D., and Carter, O. (2015). Quantitative review finds no evidence of cognitive effects in healthy populations from single-session transcranial direct current stimulation (tDCS). Brain Stimul. 8, 535-550. doi: 10.1016/j.brs.2015.01.400

Horwitz, B., Rumsey, J. M., and Donohue, B. C. (1998). Functional connectivity of the angular gyrus in normal reading and dyslexia. Proc. Natl. Acad. Sci. U.S.A. 95, 8939-8944. doi: 10.1073/pnas.95.15.8939

Kekic, M., Boysen, E., Campbell, I. C., and Schmidt, U. (2016). A systematic review of the clinical efficacy of transcranial direct current stimulation (tDCS) in psychiatric disorders. J. Psychiatr. Res. 74, 70-86. doi: 10.1016/j.jpsychires.2015.12.018

Kuo, H. I., Bikson, M., Datta, A., Minhas, P., Paulus, W., Kuo, M. F., et al. (2013). Comparing cortical plasticity induced by conventional and highdefinition $4 \times 1$ ring tDCS: a neurophysiological study. Brain Stimul. 6, 644-648. doi: 10.1016/j.brs.2012.09.010

Lawrence, B. J., Gasson, N., Johnson, A. R., Booth, L., and Loftus, A. M. (2018). Cognitive training and transcranial direct current stimulation for mild cognitive impairment in parkinson's disease: a randomized controlled trial. Parkinson's Dis. 2018:4318475. doi: 10.1155/2018/4318475

Liebetanz, D., Nitsche, M. A., Tergau, F., and Paulus, W. (2002). Pharmacological approach to the mechanisms of transcranial DC-stimulation-induced after-effects of human motor cortex excitability. Brain 125,2238-2247. doi: 10.1093/brain/awf238

Lindenberg, R., Renga, V., Zhu, L. L., Nair, D., and Schlaug, G. (2010). Bihemispheric brain stimulation facilitates motor recovery in chronic stroke patients. Neurology 75, 2176-2184. doi: 10.1212/WNL.0b013e318202013a

Martin, A., Schurz, M., Kronbichler, M., and Richlan, F. (2015). Reading in the brain of children and adults: a meta-analysis of 40 functional magnetic resonance imaging studies. Human Brain Mapp. 36, 1963-1981. doi: 10.1002/hbm.22749

Martin, D. M., Liu, R., Alonzo, A., Green, M., Player, M. J., and Sachdev, P., et al. (2013). Can transcranial direct current stimulation enhance outcomes from cognitive training? A randomized controlled trial in healthy participants. Int. J. Neuropsychopharmacol. 16, 1927-1936. doi: 10.1017/S146114571 3000539

Mccandliss, B. D., and Noble, K. G. (2003). The development of reading impairment: a cognitive neuroscience model. Dev. Disabil. Res. Rev. 9, 196-205. doi: $10.1002 / \operatorname{mrdd} .10080$

McKendrick, R., Parasuraman, R., and Ayaz, H. (2015). Wearable functional near infrared spectroscopy (fNIRS) and transcranial direct current stimulation (tDCS): expanding vistas for neurocognitive augmentation. Front. Syst. Neurosci. 9:27. doi: 10.3389/fnsys.2015.00027

Meinzer, M., Jähnigen, S., Copland, D. A., Darkow, R., Grittner, U., Avirame, K., et al. (2014). Transcranial direct current stimulation over multiple days improves learning and maintenance of a novel vocabulary. Cortex 50, 137-147. doi: 10.1016/j.cortex.2013.07.013 
Meyler, A., Keller, T. A., Cherkassky, V. L., Gabrieli, J. D., and Just, M. A. (2008). Modifying the brain activation of poor readers during sentence comprehension with extended remedial instruction: a longitudinal study of neuroplasticity. Neuropsychologia 46, 2580-2592. doi: 10.1016/j.neuropsychologia.2008.03.012

Miller, J., Berger, B., and Sauseng, P. (2015). Anodal transcranial direct current stimulation (tDCS) increases frontal-midline theta activity in the human EEG: a preliminary investigation of non-invasive stimulation. Neurosci. Lett. 588, 114-119. doi: 10.1016/j.neulet.2015.01.014

Miniussi, C., and Vallar, G. (2011). Brain stimulation and behavioural cognitive rehabilitation: a new tool for neurorehabilitation? Neuropsychol. Rehabil. 21, 553-559. doi: 10.1080/09602011.2011.622435

Monte-Silva, K., Kuo, M. F., Hessenthaler, S., Fresnoza, S., Liebetanz, D., and Paulus, W. (2013). Induction of late LTP-like plasticity in the human motor cortex by repeated non-invasive brain stimulation. Brain Stimul. 6, 424-432. doi: 10.1016/j.brs.2012.04.011

Monte-Silva, K., Kuo, M. F., Liebetanz, D., Paulus, W., and Nitsche, M. A. (2010). Shaping the optimal repetition interval for cathodal transcranial direct current stimulation (tDCS). J. Neurophysiol. 103, 1735-1740. doi: $10.1152 /$ jn. 00924.2009

Morken, F., Helland, T., Hugdahl, K., and Specht, K. (2017). Reading in dyslexia across literacy development: a longitudinal study of effective connectivity. Neuroimage 144 (Pt A), 92-100. doi: 10.1016/j.neuroimage. 2016.09.060

Nitsche, M. A., Liebetanz, D., Antal, A., Lang, N., Tergau, F., and Paulus, W. (2003). Chapter 27 modulation of cortical excitability by weak direct current stimulation - technical, safety and functional aspects. Suppl. Clin. Neurophysiol. 56, 255-276. doi: 10.1016/S1567-424X(09)70230-2

O'Connell, N. E., Cossar, J., Marston, L., Wand, B. M., Bunce, D., Moseley, G. L., et al. (2012). Rethinking clinical trials of transcranial direct current stimulation: participant and assessor blinding is inadequate at intensities of $2 \mathrm{~mA}$. PLOS ONE 7:e0047514. doi: 10.1371/journal.pone.0047514

Palm, U., Keeser, D., Schiller, C., Fintescu, Z., Nitsche, M., Reisinger, E., et al. (2008). Skin lesions after treatment with transcranial direct current stimulation (tDCS). Brain Stimul. 1, 386-387. doi: 10.1016/j.brs.2008.04.003

Papagiannopoulou, E. A., and Lagopoulos, J. (2016). Resting state EEG hemispheric power asymmetry in children with dyslexia. Front. Pediatr. 4:9830. doi: 10.3389/fped.2016.00011

Park, S. H., Seo, J. H., Kim, Y. H., and Ko, M. H. (2013). Long-term effects of transcranial direct current stimulation combined with computerassisted cognitive training in healthy older adults. Neuroreport 25, 122-126. doi: 10.1097/WNR.0000000000000080

Paulesu, E., Démonet, J. F., Fazio, F., Mccrory, E., Chanoine, V., Brunswick, N., et al. (2001). Dyslexia: cultural diversity and biological unity. Science 291, 2165-2167. doi: 10.1126/science.1057179

Penolazzi, B., Bergamaschi, S., Pastore, M., Villani, D., Sartori, G., and Mondini, S. (2015). Transcranial direct current stimulation and cognitive training in the rehabilitation of alzheimer disease: a case study. Neuropsychol. Rehabil. 25, 799-817. doi: 10.1080/09602011.2014.977301

Penolazzi, B., Spironelli, C., and Angrilli, A. (2010). Delta EEG activity as a marker of dysfunctional linguistic processing in developmental dyslexia. Psychophysiology 45, 1025-1033. doi: 10.1111/j.1469-8986.2008.00709.x

Pham, A. V., and Hasson, R. M. (2014). Verbal and visuospatial working memory as predictors of children's reading ability. Arch. Clin. Neuropsychol. 29, 467-477. doi: 10.1093/arclin/acu024

Rae,C., Arul-Anandam, P., Moffat, K., and Loo, C. K. (2009). Plugged in and Switched on: the effect of transcranial direct current stimulation in healthy controls. Proc. Int. Soc. Magnetic Resonance Med. 17:3460.

Ravizza, S. M., Becker, J. T., Delgado, M. R., Chein, J. M., and Fiez, J. A. (2002), Contribution of the inferior parietal cortex to verbal working memory. J. Cogn. Neurosci. 36:36.

Ravizza, S. M., Delgado, M. R., Chein, J. M., Becker, J. T., and Fiez, J. A. (2004). Functional dissociations within the inferior parietal cortex in verbal working memory. Neuroimage 22, 562-573. doi: 10.1016/j.neuroimage.2004. 01.039

Richards, T. L., and Berninger, V. W. (2008). Abnormal fMRI connectivity in children with dyslexia during a phoneme task: before but not after treatment. J. Neurolinguist. 21, 294-304. doi: 10.1016/j.jneuroling.2007.07.002
Richardson, J., Datta, A., Dmochowski, J., Parra, L. C., and Fridriksson, J. (2015). Feasibility of using high-definition transcranial direct current stimulation (hd-tDCS) to enhance treatment outcomes in persons with aphasia. Neurorehabilitation 36:115.

Richlan, F., Kronbichler, M., and Wimmer, H. (2010). Functional abnormalities in the dyslexic brain: a quantitative meta-analysis of neuroimaging studies. Human Brain Mapp. 30, 3299-3308. doi: 10.1002/hbm.20752

Richlan, F., Kronbichler, M., and Wimmer, H. (2011). Meta-analyzing brain dysfunctions in dyslexic children and adults. Neuroimage 56, 1735-1742. doi: 10.1016/j.neuroimage.2011.02.040

Richmond, L. L., Wolk, D., Chein, J., and Olson, I. R. (2014). Transcranial direct current stimulation enhances verbal working memory training performance over time and near transfer outcomes. J. Cogn. Neurosci. 26, 2443-2454. doi: 10.1162/jocn_a_00657

Ruf, S. P., Fallgatter, A. J., and Plewnia, C. (2017). Augmentation of working memory training by transcranial direct current stimulation (tDCS). Sci. Rep. 7:876. doi: 10.1038/s41598-017-01055-1

Sadleir, R. J., Vannorsdall, T. D., Schretlen, D. J., and Gordon, B. (2010). Transcranial direct current stimulation (tDCS) in a realistic head model. Neuroimage 51, 1310-1318. doi: 10.1016/j.neuroimage.2010.03.052

Schulz, E., Maurer, U., van der Mark, S., Bucher, K., Brem, S., Martin, E., et al. (2008). Impaired semantic processing during sentence reading in children with dyslexia: combined fmri and ERP evidence. Neuroimage 41, 153-168. doi: 10.1016/j.neuroimage.2008.02.012

Simos, P. G., Fletcher, J. M., Sarkari, S., Billingsley, R. L., Denton, C., and Papanicolaou, A. C. (2007). Altering the brain circuits for reading through intervention: a magnetic source imaging study. Neuropsychology 21, 485-496. doi: 10.1037/0894-4105.21.4.485

Siok, W. T., Perfetti, C. A., Jin, Z., and Tan, L. H. (2004). Biological abnormality of impaired reading is constrained by culture. Nature $431,71-76$. doi: 10.1038 /nature 02865

Sklar, B., Hanley, J., and Simmons, W. W. (1972). An EEG experiment aimed toward identifying dyslexic children. Nature 240, 414-416. doi: $10.1038 / 240414 \mathrm{a} 0$

Spironelli, C., Penolazzi, B., and Angrilli, A. (2008). Dysfunctional hemispheric asymmetry of theta and beta EEG activity during linguistic tasks in developmental dyslexia. Biol. Psychol. 77, 123-131. doi: 10.1016/j.biopsycho.2007.09.009

Staels, E., and Van den Broeck, W. (2017). A specific implicit sequence learning deficit as an underlying cause of dyslexia? investigating the role of attention in implicit learning tasks. Neuropsychology 31:4. doi: 10.1037/neu0000348

Stagg, C. J., Best, J. G., Stephenson, M. C., O'Shea, J., Wylezinska, M., Kincses, Z. T., et al. (2009). Polarity-sensitive modulation of cortical neurotransmitters by transcranial stimulation. J. Neurosci. 29, 5202-5206. doi: 10.1523/JNEUROSCI.4432-08.2009

Stagg, C. J., and Nitsche, M. A. (2011). Physiological basis of transcranial direct current stimulation. Neuroscientist 17, 37-53. doi: 10.1177/1073858 410386614

Swanson, H. L., and Howell, M. (2001). Working memory, short-term memory, and speech rate as predictors of children's reading performance at different ages. J. Educ. Psychol. 93, 720-734. doi: 10.1037/0022-0663.93.4.720

Temple, E., Deutsch, G. K., Poldrack, R. A., Miller, S. L., Tallal, P., and Merzenich, M. M. (2003). Neural deficits in children with dyslexia ameliorated by behavioral remediation: evidence from functional MRI. Proc. Natl. Acad. Sci. U.S.A. 100, 2860-2865. doi: 10.1073/pnas.0030098100

Teo, F., Hoy, K. E., Daskalakis, Z. J., and Fitzgerald, P. B. (2011). Investigating the role of current strength in tDCS modulation of working memory performance in healthy controls. Front. Psychiatry 2:45. doi: 10.3389/fpsyt.2011. 00045

Thomson, J. M., Doruk, D., Mascio, B., Fregni, F., and Cerruti, C. (2015). Transcranial direct current stimulation modulates efficiency of reading processes. Front. Hum. Neurosci. 9:114. doi: 10.3389/fnhum.2015. 00114

Turkeltaub, P. E., Benson, J., Hamilton, R. H., Datta, A., Bikson, M., and Coslett, H. B. (2012). Left lateralizing transcranial direct current stimulation improves reading efficiency. Brain Stimul. 5, 201-207. doi: 10.1016/j.brs. 2011.04.002 
Wagner, T., Fregni, F., Fecteau, S., Grodzinsky, A., Zahn, M., and Pascualleone, A. (2007). Transcranial direct current stimulation: a computer-based human model study. Neuroimage 35, 1113-1124. doi: 10.1016/j.neuroimage.2007.01.027

Westwood, S. J., Olson, A., Miall, R. C., Nappo, R., and Romani, C. (2017). Limits to tDCS effects in language: failures to modulate word production in healthy participants with frontal or temporal tDCS. Cortex 86, 64-82. doi: 10.1016/j.cortex.2016.10.016

Westwood, S. J., and Romani, C. (2017). Transcranial direct current stimulation (tDCS) modulation of picture naming and word reading: a meta-analysis of single session tDCS applied to healthy participants. Neuropsychologia 2017:S0028393217302841. doi: 10.1016/j.neuropsychologia.2017. 07.031

Younger, J. W., Randazzo Wagner, M., and Booth, J. R. (2016). Weighing the cost and benefit of transcranial direct current stimulation on different reading subskills. Front. Neurosci. 10:262. doi: 10.3389/fnins.2016.00262
Yue, Q., Martin, R. C., Hamilton, A. C., and Rose, N. S. (2018). Nonperceptual regions in the left inferior parietal lobe support phonological shortterm memory: evidence for a buffer account? Cerebral Cortex 2018, 1-16. doi: $10.1093 /$ cercor/bhy037

Conflict of Interest Statement: The authors declare that the research was conducted in the absence of any commercial or financial relationships that could be construed as a potential conflict of interest.

Copyright (c) 2019 Zhang, Song, Chen, Zuo, Xia and Zhang. This is an open-access article distributed under the terms of the Creative Commons Attribution License (CC

$B Y)$. The use, distribution or reproduction in other forums is permitted, provided the original author(s) and the copyright owner(s) are credited and that the original publication in this journal is cited, in accordance with accepted academic practice. No use, distribution or reproduction is permitted which does not comply with these terms. 